\title{
A SEARCH FOR WHITE DWARF COMPANIONS OF Be STARS
}

\author{
A.J.M. PITERS ${ }^{1)}$, E.J.A. MEURS ${ }^{2)}$, J. COTÉ ${ }^{1)}$, M.H. VAN KERKWIJK ${ }^{1)}$, \\ J. VAN PARADIJS ${ }^{1)}$, O.R. POLS ${ }^{1)}$, L.B.F.M. WATERS ${ }^{3)}$ \\ 1)Astr. Institute "Anton Pannekoek", Amsterdam, The Netherlands, \\ $\mathscr{E}$ Center for High-Energy Astroph., Amsterdam, The Netherlands \\ 2) Max Planck Institut für Extraterrestrische Physik, \\ Garching bei München, Germany \\ 3)SRON Laboratory for Space Research, Groningen, The Netherlands
}

\begin{abstract}
Preliminary results of an extended multi-wavelength study of bright B- and Betype stars are presented. This project aims at finding evidence for the existence of white-dwarf companions to Be stars which are predicted as the result of close binary evolution. Comparison of ROSAT all-sky survey data with simultaneous measurements of $\mathrm{H} \alpha$ profiles and infrared photometry suggests that there is no difference in the X-ray behaviour of Be stars with respect to that of normal B stars. The ROSAT X-ray luminosities of B and Be stars range from $10^{-8}$ to $10^{-5}$ times the bolometric luminosity.
\end{abstract}

\section{Introduction}

Evolutionary calculations of intermediate-mass close-binary systems by Pols et al. (1991) indicate the existence of a large number of Be star binaries with an evolved companion: a helium star, a white dwarf or a neutron star. The expected fractions of post-mass-transfer B stars with those types of companions were calculated on the basis of a model of case B close-binary evolution. Among the stars in the Bright Star Catalogue, about 4 Be stars with neutron star companions are expected (if we assume that all rapidly rotating $B$ stars formed by mass transfer indeed show up as Be stars). Similarly, some $20 \mathrm{Be}+$ white-dwarf systems and some $60 \mathrm{Be}+$ helium star systems are expected in this catalogue, for reasonable values of the model parameters.

$\mathrm{Be}+$ neutron star systems are identified with the well-known Be/X-ray binaries (Van den Heuvel and Rappaport, 1987; Waters, 1989). According to Waters et al. (1989), the $\mathrm{Be}+$ white dwarf binaries should also be X-ray sources, although with lower luminosities and with somewhat softer X-ray spectra. On the basis of a wind-fed accretion model, they estimated X-ray luminosities between $10^{29}$ and $10^{33} \mathrm{erg} / \mathrm{s}$. A number of candidate $\mathrm{Be}+$ white-dwarf binaries is mentioned by Waters et al. (1989) and Waters (1989).

The ROSAT all-sky survey is expected to reveal a large number low-luminosity $\mathrm{Be} / \mathrm{X}$ ray binaries. We started a multi-wavelength campaign in order to observe all Be stars, and a number of 'normal' B stars for control, in the Bright Star Catalogue simultaneously with the ROSAT survey. The simultaneity is important because of the intrinsic variability of Be stars on many time scales.

In this paper we present the results on this campaign that are available so far. In section 2 we summarize our observing campaign. In section 3 the first results are presented, and finally in section 4 we offer some preliminary conclusions. 


\section{Observations accompanying the ROSAT data}

Spectra around the $\mathrm{H} \alpha$-region for all emission line stars in the Bright Star Catalogue (BSC) of spectral type $\mathrm{O}, \mathrm{B}$ and $\mathrm{A}$, and for a number of 'normal' $\mathrm{B}$ stars were taken simultaneously with the ROSAT observations. The northern hemisphere objects were covered with the $2.2 \mathrm{~m}$ telescope at Calar Alto, Spain, the southern hemisphere ones with the $1.4 \mathrm{~m}$ Coudee Auxiliary Telescope at ESO, Chile. Dougherty et al. (1991) obtained IR-photometry for all Be stars from the BSC with declination larger than $-30^{\circ}$ at three observatories: Rothney Astrophysical Observatory, Calgary (1.5m telescope), the National Optical Astronomy Observatory, Kitt Peak (1.3m telescope) and the Mauna Kea Observatory, Hawaii $(0.6 \mathrm{~m}$ telescope). For the southern stars we have a rather incomplete set of simultaneous IR observations taken with the $1 \mathrm{~m}$ telescope at ESO. Genève photometry has been obtained for nearly all stars in our sample at Jungfraujoch (Switzerland) and at ESO (Swiss telescope). The few stars from our sample which are the most likely candidates for having a white dwarf companion (Waters et al. 1989), have also been observed with IUE. The observing time for all these data was as close as possible to the ROSAT survey observing time. The present paper is based on the $25 \%$ of the ROSAT all sky survey data that has been processed at the time of this conference.

\section{First preliminary results}

Up till now (having analysed $25 \%$ of the survey) only four stars, classified as Be stars in the BSC, could be identified as X-ray sources: 23 Tau (HR 1156, $L_{x} \approx 7 \cdot 10^{29} \mathrm{erg} / \mathrm{s}$ ), HR $3858\left(L_{x} \approx 6 \cdot 10^{29} \mathrm{erg} / \mathrm{s}\right), \mu$ Lup (HR 5683, $L_{x} \approx 10^{30} \mathrm{erg} / \mathrm{s}$ ) and $\pi$ Aqr (HR 8539, $\left.L_{x} \approx 10^{32} \mathrm{erg} / \mathrm{s}\right)$. In Table 1 we give the number of detections and non-detections for the whole sample of $\mathrm{B}$ stars (including $\mathrm{Be}$ ) and for the sub-sample of only Be stars. These samples include only those stars for which survey data were available. The detection rate is the same for both.

TABLE 1.

\begin{tabular}{l|c|c|c} 
& nr det. & nr non-det. & fraction \\
\hline B stars (BSC) & 32 & 402 & $7.4 \pm 1.4 \%$ \\
Be stars (BSC) & 4 & 49 & $8 \pm 4 \%$ \\
OBAe stars & & & \\
(BSC+Suppl) & 6 & 68 & $8 \pm 3 \%$
\end{tabular}

For 103 of the analysed stars $\mathrm{H} \alpha$-spectra are available: $\mathrm{H} \alpha$ was in emission for 51 stars and in absorption for 52 stars. The number of ROSAT detections within these two groups is 5 and 6 , respectively.

For 46 of the analysed stars IR photometry is available. Only five of them are identified as X-ray sources, two of this five have an IR colour excess. Before we are able to give any meaningful conclusions about the behaviour of the X-ray luminosity and the detection rate compared with IR data we have to wait for the rest of the all-sky survey data.

In Figure 1 we plotted $\log \left(L_{\mathrm{X}} / L_{\mathrm{bol}}\right)$ as a function of spectral type for the 32 detected B-type stars . Typical errors are $0.2 \mathrm{dex}$. The lack of stars in the lower right part 


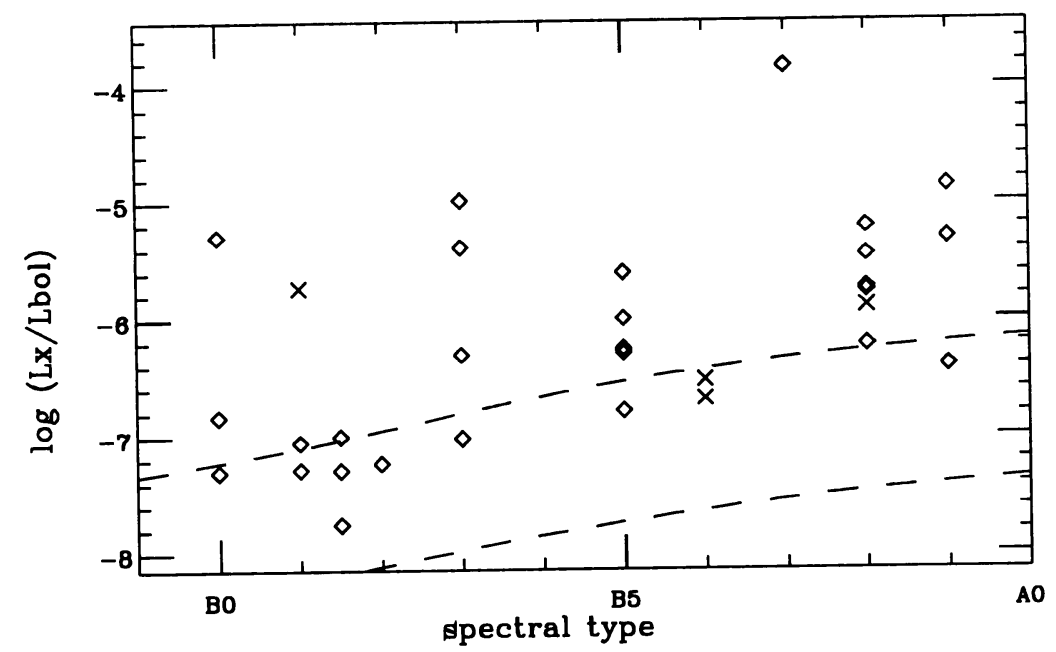

Figure 1. $\log \left(L_{\mathrm{X}} / L_{\mathrm{bol}}\right)$ as a function of spectral type. Crosses indicate emission line stars, diamonds indicate 'normal' stars. The dashed lines indicate the detection limit of the survey for stars of visual magnitude 6 (top line) and 3 (bottom line).

reflects the detection limit of ROSAT (which is typically $10^{-13} \mathrm{erg} \mathrm{sec}^{-1} \mathrm{~cm}^{-2}$ ). The Xray luminosities range from $10^{-8}$ to $10^{-5}$ times the bolometric luminosity.

\section{Discussion and Conclusions}

The fraction of Be stars detected with ROSAT is not significantly larger than that of 'normal' B stars. Also the X-ray luminosities are comparable to those for 'normal' B stars. This suggests that the X-ray producing mechanism in Be stars is not different from that of other B stars: from the detection rates alone there is no reason to believe that the $\mathrm{X}$-rays from the four detected Be stars are produced by accretion onto a white dwarf, instead it could be possible that the X-rays are generated in the 'normal' stellar wind.

In Figure 1 we can see that there is no correlation between the X-ray fraction of the total luminosity and spectral type. This is surprising since it is well known that the strength of the stellar wind decreases towards later spectral type. Friend and MacGregor (1984) showed with their stellar wind models that the mass loss and terminal velocity of a wind can be enhanced by increasing the rotational velocity of the star. The fraction of the analysed stars that is identified as an X-ray source, however, is not correlated with $v_{\text {rot }} \sin i$. The mean X-ray luminosity for OBA-type stars, as derived from Einstein data (Pallavicini et al., 1981), is $10^{-7.2}$ times the bolometric luminosity. The X-ray luminosities of B stars observed with ROSAT (Figure 1) are well above this value. The difference in X-ray energy bands for these two instruments (ROSAT: $0.1-2.4 \mathrm{keV}$; Einstein: 0.2 $2.5 \mathrm{keV}$ ) causes a systematicly higher ROSAT X-ray luminosity for stars that radiate a large portion of their X-ray flux between 0.1 and $0.2 \mathrm{keV}$. But for individual stars we find 
almost the same X-ray luminosity as derived from Einstein data (Table 2). It is therefore more likely that the difference is due to the fact that the value of $10^{-7.2} \cdot L_{\mathrm{bol}}$ is based on only $14 \mathrm{~B}$ stars (together with $16 \mathrm{O}$ stars and $5 \mathrm{~A}$ stars).

TABLE 2.

\begin{tabular}{c|c|c} 
name & $\begin{array}{c}\log (\mathrm{Lx} / \mathrm{Lbol}) \\
\text { ROSAT }\end{array}$ & $\begin{array}{c}\log (\mathrm{Lx} / \mathrm{Lbol}) \\
\text { Einstein }\end{array}$ \\
\hline HR 936 & -5.1 & -5.2 \\
HR3117 & -6.9 & -7.6 \\
HR5267 & -7.3 & -7.3 \\
HR5469 & -7.7 & -7.8
\end{tabular}

The binary system HR927+HR928 (B7V+B7V) has an X-ray luminosity of approximately $10^{32} \mathrm{erg} / \mathrm{sec}$, which is $10^{-4}$ times the bolometric lominosity of a B7V star. It is conceivable that this system has interacting stellar winds, which cause shocks in the region of interaction, that heat the wind and generate $\mathrm{X}$-rays.

\section{references}

Dougherty, S.M., Taylor, A.R., and Clark, T.A. (1991), Astron. J., in press

Friend, D.B., and MacGregor, K.B. (1984), Astrophys. J. 282, 591

van den Heuvel, E.P.J., and Rappaport, S. (1987), in Proc. IAU Symposium 98,

"Physics of Be Stars", Eds. A. Slettebak and T.P. Snow, p. 291

Pallavicini, R., Golub, L., Rosner, R., Vaiana, G.S., Ayres, T., and Linsky, J.L. (1981), Astrophys. J. 248, 279

Pols, O.R., Coté, J., Waters, L.B.F.M., and Heise, J. (1991), Astron. Astroph. 241, 419

Waters, L.B.F.M. (1989), in: Proceedings of the 23rd ESLAB Symposium, ESA SP-296 (Vol. 1), p. 25

Waters, L.B.F.M., Pols, O.R., Hogeveen, S.J., Coté, J., van den Heuvel, E.P.J. (1989), Astron. Astrophys. 220, L1 